Bundesgesundheitsbl 2012 · 55:1444-1452

DOI 10.1007/s00103-012-1555-7

Online publiziert: 21. Oktober 2012

(c) Springer-Verlag 2012

\section{Ruscher ${ }^{1} \cdot$ R. Schaumann ${ }^{2} \cdot$ M. Mielke ${ }^{1}$}

${ }^{1}$ Abteilung für Infektionskrankheiten, Fachgebiet 14 (Angewandte Infektions-

und Krankenhaushygiene), Robert Koch-Institut, Berlin

${ }^{2}$ Institut für Medizinische Mikrobiologie und Infektionsepidemiologie, Universität Leipzig

\title{
Herausforderungen durch Infektionen und mehrfach- resistente Bakterien bei alten Menschen in Heimen
}

Das Infektionsschutzgesetz (IfSG) definiert die „nosokomiale Infektion“ als eine Infektion mit lokalen oder systemischen Infektionszeichen als Reaktion auf das Vorhandensein von Erregern oder ihrer Toxine, die im zeitlichen $\mathrm{Zu}$ sammenhang mit einer stationären oder ambulanten medizinischen Maßnahme steht, soweit die Infektion nicht bereits vorher bestand. Diese Definition macht deutlich, dass der oft synonym verwendete Begriff „Krankenhausinfektion“ $\mathrm{zu}$ eng gefasst ist. Er grenzt solche Infektionen aus, die sich in über 11.000 Altenund Pflegeheimen und im Rahmen ambulanter Pflege durch rund 11.000 Pflegedienste ereignen können. Es sind in der Mehrzahl alte Menschen, die in diesen Einrichtungen betreut oder durch die ambulanten Dienste gepflegt werden. Die Verbreitung multiresistenter Erreger und die daraus entstehenden therapeutischen Limitationen führen zu Fragen, denen wir uns im Robert Koch-Institut täglich stellen müssen. Der vorliegende Beitrag soll helfen, die Dimension des Problems nosokomialer Infektionen und multiresistenter Erreger bei Menschen mit altersbedingter Multimorbidität aufzuzeigen. Dabei stellen sich zunächst folgende Fragen:

- Wie viele Menschen leben in Deutschland im Alter in Pflegeeinrichtungen?

- Welche Bedeutung haben Infektionen bei alten Menschen im Allgemeinen und bei alten Menschen, die in Pflegeeinrichtungen leben, im Speziellen?
- Welche Rolle spielen dabei die Anwendung von Antibiotika und multiresistente Erreger (MRE)? Wie häufig kommen MRE bei alten Menschen in Pflegeeinrichtungen vor?

- Wie wird in Pflegeeinrichtungen mit alten Menschen umgegangen, die mit multiresistenten Erregern kolonisiert oder infiziert sind und welche Konsequenzen ergeben sich für die $\mathrm{Zu}$ kunft?

\section{Demografie}

Sinkende Geburtenraten und die steigende Lebenserwartung der Menschen in Deutschland führen in den kommenden Jahren und Jahrzehnten nicht nur zu einem Rückgang der Gesamtbevölkerungszahl, sondern auch zu einem drastischen Wandel in der Altersstruktur. Den Prognosen der statistischen Ämter des Bundes und der Länder zufolge wird im Jahr 2030 mehr als jeder dritte Einwohner in Deutschland zu den über 60-Jährigen zählen [1].

Da vor allem ältere Menschen chronische Erkrankungen haben und pflegebedürftig sind, wird auch der Anteil pflegebedürftiger Menschen in Deutschland zunehmen. Innerhalb der Bevölkerung vollzieht sich somit eine Verschiebung hin zu den Altersgruppen, die ein höheres Risiko haben, pflegebedürftig zu sein oder in Folge einer Krankheit stationär behandelt werden zu müssen. Dem Bedarf an Versorgung und Pflege alter Menschen gerecht zu werden wird vermutlich eine der großen gesellschaftspolitischen Herausforderungen dieses Jahrhunderts sein.

Ende 2009 waren der deutschen Pflegestatistik zufolge insgesamt 2,34 Mio. Menschen pflegebedürftig. Knapp ein Drittel davon $(717.490 ; 31 \%)$ wurde in Heimen versorgt. Bei einer deutschen Gesamtbevölkerungszahl von 81,88 Mio. Menschen im Jahr 2009 entspricht der Anteil der in Heimen versorgten Pflegebedürftigen einem knappen Prozent der Gesamtbevölkerung (0,9\%; [2]). Die wichtigsten Kennzahlen zur stationären Pflege in Deutschland sind in $\bullet$ Tab. 1 zusammengefasst.

Verglichen mit der Betreuung durch Angehörige oder ambulante Pflegedienste im häuslichen Lebensumfeld älterer Menschen findet in der stationären Pflege wesentlich häufiger die Versorgung sehr umfänglich pflegebedürftiger Menschen statt (- Tab. 2). Dies ist neben dem hohen Lebensalter der Heimbewohner nicht zuletzt auch der relativ frühen (Rück-)Verlegung von Patienten aus dem Krankenhaus in Pflegeheime geschuldet. Betrachtet man den Übergang der Menschen in das Pflegeheim etwas genauer, so erkennt man, dass ein nicht unerheblicher Teil von ihnen unmittelbar aus einem Akutkrankenhaus dauerhaft in das Pflegeheim verlegt wird. Daten einer retrospektiven Kohortenstudie aus Deutschland zufolge wurden $45 \%$ der Heimbewohner direkt vom Krankenhaus in das Pflegeheim überwiesen; bei weiteren $15 \%$ lag der letzte Krankenhausaufenthalt vor Heimeintritt nicht länger als 4 Wochen zurück [3]. 
Tab. 1 Kennzahlen zur stationären Pflege in Deutschland (Daten der Pflegestatistik 2009; Statistisches Bundesamt, Wiesbaden; Erster Bericht des Bundesministeriums für Familie, Senioren, Frauen und Jugend über die Situation der Heime und die Betreuung der Bewohnerinnen und Bewohner 2006; Bundesministerium für Familie, Senioren, Frauen und Jugend; Gesundheitsberichterstattung des Bundes)

\begin{tabular}{|ll}
\hline Anzahl Pflegebedürftiger insgesamt & 2,34 Mio. \\
\hline Pflegequote (alle Altersstufen) & $2,9 \%$ \\
\hline Männer & $1,9 \%$ \\
\hline Frauen & $3,8 \%$ \\
\hline Davon stationär in Heimen versorgt & 717.490 (31\%) \\
\hline Entspricht einem Anteil der deutschen Gesamtbevölkerung 2009 von & $0,9 \%$ \\
\hline Anteil der über 60-jährigen & 682.965 (95\%) \\
\hline Frauenanteil & 538.117 (75\%) \\
\hline Anteil schwer pflegebedürftiger Menschen (Pflegestufe III) & 146.835 (20,5\%) \\
\hline Anzahl voll- und teilstationärer Pflegeheime & 11.600 \\
\hline Durchschnittliche Anzahl pro Heim betreuter Pflegebedürftiger & 64 \\
\hline Durchschnittliches Alter bei Eintritt in die Pflegeeinrichtung & 81 Jahre \\
\hline Durchschnittliche Verweildauer in der Einrichtung & 41,3 Monate (3,4 Jahre) \\
\hline Männer & 25,9 Monate (2,2 Jahre) \\
\hline Frauen & 47,4 Monate (3,9 Jahre) \\
\hline Durchschnittliches Lebensalter in der Einrichtung & 81,8 Jahre \\
\hline Männer & 76,8 Jahre \\
\hline Frauen & 83,6 Jahre \\
\hline Durchschnittliches Sterbealter & 85,3 Jahre \\
\hline Männer & 79,3 Jahre \\
\hline Frauen & 87,6 Jahre \\
\hline
\end{tabular}

Tab. 2 Versorgungsarten bei Pflegebedürftigkeit und schwerer Pflegebedürftigkeit ${ }^{\mathrm{a}}$ in den Jahren 1999, 2005 und 2009. Daten der deutschen Pflegestatistik 1999, 2005, 2009 (Statistisches Bundesamt, Deutschlandergebnisse)

\begin{tabular}{|c|c|c|c|c|c|c|c|}
\hline \multirow[t]{2}{*}{ Versorgungsart } & \multicolumn{2}{|l|}{1999} & \multicolumn{2}{|l|}{2005} & \multicolumn{2}{|l|}{2009} & \multirow{2}{*}{$\begin{array}{l}\text { Veränderungen } \\
1999 \text { zu } 2009 \text { (\%) } \\
\text { Pflegebedürf- } \\
\text { tige insgesamt } \\
\text { (Anzahl) }\end{array}$} \\
\hline & $\begin{array}{l}\text { Pflegebedürftige } \\
\text { insgesamt } \\
\text { (Anzahl) }\end{array}$ & $\begin{array}{l}\text { Anteil } \\
\text { Pflege- } \\
\text { stufe III } \\
(\%)\end{array}$ & $\begin{array}{l}\text { Pflege- } \\
\text { bedürftige } \\
\text { insgesamt } \\
\text { (Anzahl) }\end{array}$ & $\begin{array}{l}\text { Anteil } \\
\text { Pflege- } \\
\text { stufe III } \\
\text { (\%) }\end{array}$ & $\begin{array}{l}\text { Pflege- } \\
\text { bedürftige } \\
\text { insgesamt } \\
\text { (Anzahl) }\end{array}$ & $\begin{array}{l}\text { Anteil } \\
\text { Pflege- } \\
\text { stufe III } \\
(\%)\end{array}$ & \\
\hline $\begin{array}{l}\text { Pflege zu Hause durch Angehörige } \\
\text { (Pflegegeldempfänger) }\end{array}$ & 1.030 .000 & 10 & 980.000 & 8,3 & 1.065 .564 & 7,7 & $+35.564(3,45 \%)$ \\
\hline Pflege durch ambulante Pflegedienste & 415.000 & 14 & 472.000 & 12,4 & 555.000 & 11,6 & $+140.000(33,73 \%)$ \\
\hline Pflegebedürftige vollstationär im Heim & 573.000 & 22 & 677.000 & 20,9 & 717.000 & 20,5 & $+144.000(25,13 \%)$ \\
\hline
\end{tabular}

Eine andere deutsche Erhebung konnte zeigen, dass es im Verlauf eines Jahres bei 231 Heimbewohnern zu insgesamt 245 stationären Aufnahmen in eine geriatrische Klinik kam mit einer durchschnittlichen Verweildauer in der Klinik von gut 32 Tagen [4].

\section{Infektionen bei alten Menschen}

Im Vergleich zu jungen Erwachsenen zeichnen sich ältere Menschen durch eine erhöhte Disposition gegenüber Infektionskrankheiten aus. Der Alterungsprozess ist assoziiert mit einer Schwächung der Immunität, die sowohl die unspezifische Abwehr (z. B. durch verminderte Speichelsekretion oder Anstieg des Magensäure-pH-Wertes) als auch die spezifische Abwehr (z. B. durch funktionelle Veränderungen der T-Zellen und B-Zellen) betrifft [5]. Die Veränderungen der spezifischen Abwehr in Form einer verminderten Antikörperproduktion und TZell-Proliferation erklären das im Alter zu beobachtende verminderte Ansprechen auf Impfungen bzw. auch die Reaktivierung einer Tuberkulose oder Varizelleninfektion (Zoster). Während dieser Prozess der „Immunoseneszenz“, also der al- tersbedingten Dysfunktion der Immunantwort, bei gesunden älteren Menschen meist eine geringe klinische Relevanz hat, kann es beim Zusammenspiel mit zusätzlichen Faktoren wie bei chronischen Erkrankungen (z. B. Neoplasien) oder auch bei einer Mangelernährung zur weiteren Schwächung der Infektabwehr kommen [5, 6]. Zudem erhöhen Infektionen bei älteren Menschen die Morbidität und Letalität. So ist die Sterblichkeit durch Infektionen des Urogenitaltraktes, des unteren Respirationstraktes, des Haut- und Weichteilgewebes sowie durch bakterielle Meningitiden und Endokarditiden bei 
älteren Menschen im Schnitt 3-mal höher als bei jungen Menschen [7]. Auch beeinflussen anatomisch-physiologische Veränderungen im Alter die Disposition gegenüber Infektionskrankheiten. Eine herabgesetzte mukoziliäre Clearance und Schluckstörungen können z. B. die Entstehung von Pneumonien begünstigen [8]. Auch im Urogenitaltrakt begünstigen altersbedingte mechanische Veränderungen wie eine reduzierte Blasenkapazität, ein verminderter Urinfluss und in der Blase verbleibender Residualurin sowie hormonelle Veränderungen in der Postmenopause und Veränderungen des Urothels eine mikrobielle Besiedlung und die Entstehung von Infektionen [9]. Pneumonien, Bakteriämien und Infektionen durch Influenzaviren gehören zu den 10 häufigsten Todesursachen bei alten Menschen [7].

Auch zeigt die klinische Manifestation von Infektionskrankheiten bei älteren Menschen oftmals spezifische Besonderheiten, die nicht selten die Diagnostik und damit eine schnelle und gezielte Therapie erschweren. Fieber als Leitsymptom bakterieller Infektionen wird oft gar nicht beobachtet. Atypische Manifestationen wie allgemeine Schwäche, Anorexie oder auch Delirium und Konfusion kommen häufig vor, zeigen sich in der Geriatrie allerdings sehr häufig auch vollkommen unabhängig von infektiösen Erkrankungen [10]. Die häufigsten Infektionen alter Menschen gehen auf pyogene Bakterien zurück. Das Spektrum der am häufigsten diagnostizierten Infektionen des alten Menschen schließt daher insbesondere Infekte des Urogenitaltraktes, Pneumonien, intraabdominale Infektionen (Divertikulitiden, Cholezystitis und Appendizitis) Endokarditiden, Bakteriämien sowie Haut- und Weichgewebsinfekte (hier vor allem der diabetische Fuß, Dekubitalulzera und Ulcus cruris) ein [11]. In Studien über Bewohner von Langzeitpflegeeinrichtungen, die im Verlauf ihres Aufenthaltes eine Sepsis entwickelten, wurde gezeigt, dass sich in $70-80 \%$ der Fälle die Sepsis infolge von Harnwegsinfekten, Atemwegsinfekten bzw. Haut- und Weichgewebsinfektionen entwickelte [12]. Besondere Bedeutung haben symptomatische Harnwegsinfektionen, da sie nicht nur zu den häufigsten bakteriellen Infek-

Bundesgesundheitsbl 2012 ·55:1444-1452 DOI 10.1007/s00103-012-1555-7

(c) Springer-Verlag 2012

\section{Ruscher $\cdot$ R. Schaumann $\cdot$ M. Mielke}

\section{Herausforderungen durch Infektionen und mehrfach-resistente Bakterien bei alten Menschen in Heimen}

\section{Zusammenfassung}

Nosokomiale Infektionen betreffen nicht nur Patienten, die im Rahmen von Krankenhausaufenthalten behandelt werden, sondern auch Menschen, die im Bereich der stationären und ambulanten Pflege versorgt werden. Da dies in der Regel ältere Menschen sind, birgt der demografische Wandel in Deutschland eine Vielzahl neuer Herausforderungen im Bereich der Infektionsprävention. Der Alterungsprozess des Menschen geht mit einigen physiologischen Veränderungen einher, die die individuelle Disposition gegenüber Infektionskrankheiten erhöhen können. Für alte Menschen, die in Heimen leben, ergeben sich durch den Gemeinschaftsalltag, durch pflegerische Maßnahmen und den teils inadäquaten Einsatz von Antibiotika mitunter zusätzliche Infektionsrisiken. Bakterien, die Resistenzen gegenüber gebräuchlichen antimi- krobiellen Wirkstoffen erworben haben, sind auch bei Bewohnern von Pflegeeinrichtungen inzwischen verbreitet. Die Einrichtungen müssen sich im Rahmen ihrer Präventionskonzepte der Aufgabe stellen, eine geeignete Balance zwischen der Wahrung des häuslichen Lebensumfelds und dem Infektionsschutz zu finden, und fühlen sich hierbei vielfach allein gelassen. Der vorliegende Beitrag soll einen Einblick geben in die Charakteristika der Infektionen des alten Menschen, insbesondere im Hinblick auf die stationäre Pflege.

\section{Schlüsselwörter}

Alte Menschen · Prävalenz .

Mehrfach-resistente Bakterien .

Antibiotikaeinsatz · Infektionsprävention

\section{The challenge of infections and multiresistant bacteria among the elderly living in long-term care facilities}

\section{Abstract}

Healthcare-associated infections not only affect patients in acute care hospitals but also patients in need of long-term care. As the elderly are generally most affected, the demographic change in Germany faces a range of increasing challenges in the field of infection control. The ageing process itself is accompanied by several physiological and pathological changes which may result in an increase in the risk of infectious diseases. Elderly living in long-term care facilities (LTCFs) may in addition be exposed to further risks due to their everyday life in a community, nursing care and the, to some extent, inappropriate use of antibiotics. Bacteria that have become resis- tant to commonly used antimicrobial agents are meanwhile prevalent in nursing homes. Caregivers often feel left alone when facing the task of achieving a balance between the need for a comfortable familiar environment and the application of infection control measures according to a resolute prevention strategy. This review aims to give an overview about the characteristics of infections among the elderly, especially with respect to longterm care.

\section{Keywords}

Elderly people $\cdot$ Prevalence $\cdot$ Multiresistant bacteria · Antibiotics use · Infection control tionen bei alten Menschen zählen, sondern auch eine der häufigsten Indikationen für das Verschreiben antimikrobieller Wirkstoffe bei ihnen sind [13]. Neben dieser Erkrankung ist aber auch die asymptomatische Bakteriurie eine häufige Diagnose. Bei Altenheimbewohnerinnen liegt die Prävalenz bei $25-50 \%$, bei Patientinnen mit harnableitenden Blasenkathetern werden nicht selten auch deutlich höhere Prävalenzen festgestellt [9].

Das Erregerspektrum typischer Infektionen bei alten Menschen unterschei- det sich von dem bei jungen Erwachsenen. Während Harnwegsinfekte bei jungen Erwachsenen vorwiegend eine durch Escherichia coli und Staphylococcus saprophyticus verursachte Erkrankung junger Frauen sind, steigt die Inzidenz im Verlauf des Alters sowohl bei Männern als auch bei Frauen. Allerdings sind hier häufig andere gramnegative Bakterien (Proteus spp., Klebsiella spp., Enterobacter spp, Pseudomonas aeruginosa) als Infektionserreger zu isolieren, während S. saprophyticus in dieser Population typischerweise 
Tab. 3 Prävalenzstudien zum Vorkommen von Methicillin-resistenten Staphylococcus aureus (MRSA) in Langzeitpflegeeinrichtungen

\begin{tabular}{|c|c|c|c|c|c|c|c|c|}
\hline \multirow[t]{2}{*}{ Autoren } & \multirow[t]{2}{*}{ Jahr } & \multirow[t]{2}{*}{ Nation } & \multirow[t]{2}{*}{ Studiendesign } & \multirow[t]{2}{*}{ Probenmaterial } & \multirow{2}{*}{$\begin{array}{l}\text { Anzahl } \\
\text { der } \\
\text { Heime }\end{array}$} & \multirow{2}{*}{$\begin{array}{l}\text { Anzahl der } \\
\text { untersuchten } \\
\text { Bewohner }\end{array}$} & \multicolumn{2}{|c|}{ Prävalenz } \\
\hline & & & & & & & (\%) & $\mathbf{n}$ \\
\hline $\begin{array}{l}\text { Pfingsten-Würzburg } \\
\text { et al. [41] }\end{array}$ & 2011 & Deutschland & Punktprävalenzstudie & $\begin{array}{l}\text { Nasentupfer; Wundtupfer, sofern } \\
\text { vorhanden }\end{array}$ & 32 & 1827 & 7,6 & 139 \\
\hline Woltering et al. [38] & 2008 & Deutschland & Punktprävalenzstudie & $\begin{array}{l}\text { Nasen-Rachen-Tupfer; Wundtupfer, } \\
\text { sofern vorhanden }\end{array}$ & 6 & 265 & 2,3 & 6 \\
\hline Neuhaus et al. [40] & 2002 & Deutschland & Querschnittsstudie & $\begin{array}{l}\text { Nasen-Rachen-Tupfer; Wundtupfer, } \\
\text { sofern vorhanden }\end{array}$ & 61 & 1057 & 3 & 32 \\
\hline Von Baum et al. [37] & 2002 & Deutschland & Punktprävalenzstudie & $\begin{array}{l}\text { Nasentupfer; Wundtupfer, sofern } \\
\text { vorhanden }\end{array}$ & 47 & 3236 & 1,1 & 36 \\
\hline Heuck et al. & 2000 & Deutschland & Punktprävalenzstudie & $\begin{array}{l}\text { Nasen-Rachen-Tupfer; Wundtupfer, } \\
\text { sofern vorhanden }\end{array}$ & 31 & 1342 & 2,4 & 32 \\
\hline Greenland et al. [43] & 2011 & Niederlande & Punktprävalenzstudie & Nasentupfer & 26 & 1268 & 0,3 & 3 \\
\hline Reynolds et al. [42] & 2011 & USA & Punktprävalenzstudie & Nasentupfer & 10 & 1000 & 30,7 & 307 \\
\hline Manzur et al. & 2008 & Spanien & Querschnittsstudie & $\begin{array}{l}\text { Nasentupfer; Wundtupfer, sofern } \\
\text { vorhanden }\end{array}$ & 9 & 1377 & 16,8 & 231 \\
\hline Barr et al. & 2007 & Großbritannien & Punktprävalenzstudie & Nasentupfer & 39 & 715 & 22 & 159 \\
\hline Cretnik et al. & 2005 & Slowenien & $\begin{array}{l}\text { Wiederholte Punkt- } \\
\text { prävalenzstudie }\end{array}$ & $\begin{array}{l}\text { Nasen-Rachen-Tupfer; Wundtupfer, } \\
\text { sofern vorhanden; ggf. Achsel-/ } \\
\text { Leistenabstriche }\end{array}$ & 1 & $\begin{array}{l}107 \\
91\end{array}$ & $\begin{array}{l}9,3 \\
8,8\end{array}$ & $\begin{array}{l}10 \\
8\end{array}$ \\
\hline O'Sullivan et al. & 2000 & Irland & $\begin{array}{l}\text { Wiederholte Punkt- } \\
\text { prävalenzstudie }\end{array}$ & $\begin{array}{l}\text { Nasen-Rachen-Tupfer, Abstriche } \\
\text { von Haaransatz, Achsel, Leiste, Peri- } \\
\text { neum; Wunden sofern vorhanden }\end{array}$ & 6 & $\begin{array}{l}754 \\
743\end{array}$ & $\begin{array}{l}8,6 \\
10,1\end{array}$ & $\begin{array}{l}65 \\
75\end{array}$ \\
\hline
\end{tabular}

nicht an Harnwegsinfektionen beteiligt ist [13]. Auch bei der ambulant erworbenen Pneumonie unterscheidet sich das Spektrum der bei jungen Erwachsenen isolierten Erreger (Mycoplasma pneumoniae, Streptococcus pneumoniae, Chlamydia pneumoniae) von dem bei älteren Patienten. Hier umfasst das Keimspektrum zu einem größeren Anteil auch Haemophilus influenzae, S. aureus und gramnegative Bakterien [14].

\section{Infektionsrisiko bei alten Menschen, die in Pflegeeinrichtungen leben}

Versteht man die Entstehung von Infektionen als Wechselspiel zwischen individueller Disposition auf der einen und Exposition durch die Umgebung auf der anderen Seite, so ergibt sich für Bewohner von Altenpflegeeinrichtungen ein sich aus beiden Faktoren nährendes erhöhtes Infektionsrisiko. Aufseiten der individuellen Disposition münden bei Bewohnern von Altenpflegeeinrichtungen die eingangs erwähnten Faktoren der altersbedingten Beeinträchtigung der Infektabwehr und anatomisch physiologischen Veränderungen gemeinsam mit dem sehr hohen Lebensalter und der häufig frühen Entlassung aus dem Krankenhaus in die Langzeitpflege nicht selten in eine schwere Pflegebedürftigkeit. Eine europäische Erhebung zu Infektionen in Langzeitpflegeeinrichtungen lieferte kürzlich auch Daten zu Indikatoren der Pflegebedürftigkeit von 6496 Bewohnern deutscher Langzeitpflegeeinrichtungen. Demnach ist knapp die Hälfte der Bewohner älter als 85 Jahre. Mehr als die Hälfte ist desorientiert bzw. kognitiv beeinträchtigt (56,7\%). Knapp drei Viertel sind inkontinent, 10,2\% haben Harnwegskatheter, und 44,8\% sind in ihrer Mobilität beeinträchtigt [15]. Diese Beeinträchtigungen erhöhen die individuelle Disposition gegenüber Infektionen und damit auch das Risiko für nosokomiale Infektionen, insbesondere für solche, die mit hoher Morbidität und Mortalität einhergehen [16]. Eine deutsche Studie hat zudem bei Bewohnern eines Pflegeheims durchschnittlich 3 Begleiterkrankungen, zumeist kardiovaskulärer bzw. neurologisch/psychiatrischer Natur ermittelt [17].

Neben der veränderten individuellen Disposition kommt in Langzeitpflegeeinrichtungen aber auch der Exposition bzw. der Transmission von Erregern eine besondere Bedeutung zu. Bewohner eines Heims teilen die Versorgung mit Lebensmitteln und medizinische Pflegefaktoren, die im Rahmen von Infektionsausbrü- chen vielfach als Quelle identifiziert wurden [18]. Andererseits müssen Seniorenresidenzen bzw. Altenpflegeheime neben dem Anspruch auf medizinische Versorgung und Pflege vor allem auch dem Aspekt der sozialen Betreuung und langfristigen Wahrung der Lebensqualität ihrer Bewohner gerecht werden. Schon aufgrund dieses Aufgabenspektrums unterscheiden sich Altenpflegeeinrichtungen im Hinblick auf die Infektionsprävention und -kontrolle deutlich von Krankenhäusern oder Rehabilitationseinrichtungen. Gruppenaktivitäten tragen entscheidend zum Erhalt und zur Förderung geistiger und körperlicher Fähigkeiten sowie zum Wohlbefinden der Bewohner bei, bergen aber auch das Potenzial für Übertragungen von Infektionserregern zwischen den Bewohnern.

Je höher die individuelle Beeinträchtigung auf körperlicher und/oder geistiger Ebene ist, desto enger ist der Kontakt zu Pflegepersonal und Pflegeutensilien. Dies kann zur Transmission von Erregern beitragen sowie über invasive therapeutische oder diagnostische Maßnahmen den Erregern geeignete Eintrittspforten verschaffen. Weiterhin zeichnet sich die bakterielle Flora von Altenheimbewohnern durch Besonderheiten im Hin- 
blick auf ihre Diversität und antimikrobielle Resistenz aus. Eine Studie zum Erregerspektrum in verschiedensten Untersuchungsmaterialien (Urin, Wundabstriche, Nasentupfer, Stuhlproben, Abstriche von PEG-Sonden und Tracheostomata) von Altenheimbewohnern zeigte, dass insbesondere gramnegative Bakterien (68\%), speziell Pseudomonas aeruginosa und eine Vielzahl von Enterobakterienspezies (P. mirabilis, E. coli, K. pneumoniae) häufig zu isolieren sind. Das Spektrum grampositiver Bakterien wurde weitgehend von Enterokokken und S. aureus dominiert [19]. Zudem werden bei alten Menschen, die in Pflegeheimen leben, häufiger resistente Bakterien isoliert als bei alten Menschen, die sich zu Hause selbst versorgen [20]. Eine weitere Studie, die die bakterielle Flora bei Pflegeheimbewohnern untersuchte, konnte zeigen, dass deren Quantität und Resistenzspektrum maßgeblich vom Hygienestandard und von personellen sowie organisatorischen Voraussetzungen der Einrichtungen abhängt [21]. Die Ursachen für die Unterschiede in der bakteriellen Kolonisation bei Altenheimbewohnern sind jedoch multifaktoriell und lassen sich nicht ausschließlich durch naheliegende Kriterien wie mangelhafte Händehygiene des Pflegepersonals sowie den Selektionsdruck durch den häufigen Einsatz antimikrobieller Wirkstoffe in den Einrichtungen erklären. Vielmehr scheinen die altersbedingten Veränderungen der Immunität, eine zugrunde liegende Komorbidität, ein mitunter schlechter Ernährungszustand, funktionelle Beeinträchtigungen der Bewohner sowie invasive Devices wie Ernährungssonden und Harnwegskatheter einen geeigneten Hintergrund für eine in der Qualität und Quantität veränderte bakterielle Flora zu schaffen. Exemplarisch lässt sich dies anhand der Besiedlung von Pflegeheimbewohnern mit $S$. aureus beleuchten. Eine amerikanische Studie konnte zeigen, dass die Besiedlungsrate mit S. aureus [Methicillin-sensibel (MSSA) und/oder Methicillin-resistent (MRSA)] der Bewohner von insgesamt 14 Pflegeheimen mit $62 \%$ deutlich über der der Allgemeinbevölkerung lag. Ein signifikanter Unterschied bestand zwischen Bewohnern mit ,invasiven Devices" und solchen ohne Devices, und zwar nicht nur mit Blick auf die
Häufigkeit, sondern auch auf die Kolonisationsorte. Im Vergleich zu Bewohnern ohne Devices ließ sich bei Bewohnern mit Devices $S$. aureus nicht nur nasal und aus dem Oropharynx, sondern wesentlich häufiger auch inguinal bzw. perianal nachweisen [22]. Ähnliches konnte kürzlich auch für die Kolonisation mit Ciprofloxacin-resistenten gramnegativen Bakterien bei Pflegeheimbewohnern mit invasiven Devices gezeigt werden [23].

Ein weiteres Spezifikum mit Blick auf das Infektionsrisiko bei alten Menschen in Heimen liegt in ihrer individuellen Versorgung durch die eigenen Hausärzte. Erhebungen hierzu zeigen, dass innerhalb einer Einrichtung nicht selten 20 oder mehr Hausärzte die Bewohner betreuen [15]. Dadurch ist mitunter nicht nur die Koordination der Ärzte untereinander bei einrichtungsspezifischen Infektionspräventionskonzepten erschwert, sondern auch die von Haus- und Fachärzten bei der Behandlung des einzelnen Bewohners. Sowohl Ärzte als auch Pflegepersonal klagen zudem nicht selten über Missstände beim Informationsaustausch zwischen Ärzten und Pflegepersonal und bei der Dokumentation von Diagnosen und Therapien. Einige Studien konnten zeigen, dass bei Pflegeheimbewohnern häufig eine Vielzahl unterschiedlicher Medikamente gleichzeitig eingesetzt wird und sich unerwünschte Arzneimittelwirkungen in dieser Population häufen [24]. Im Durchschnitt erhält ein Heimbewohner 6 verschiedene Medikamente, mehr als $20 \%$ der Heimbewohner sogar 10 oder mehr [25].

\section{Häufigkeit von Infektionen \\ in Pflegeeinrichtungen \\ für alte Menschen}

Internationale Daten geben Hinweis darauf, dass Infektionen eine der Hauptursache für Morbidität und Letalität alter Menschen in Langzeitpflegeeinrichtungen sind und einen wesentlichen Anteil der Krankenhauseinweisungen ausmachen [26, 27, 28]. Ältere Studien aus den Vereinigten Staaten schätzen, dass die Letalität hier zwischen 0,04 und 0,71 pro 1000 Bewohnertage liegt. Pneumonien sind in allen Studien die führende Todesursache [29]. Anders als in Krankenhäu- sern, die durch eine entsprechende Verankerung im Infektionsschutzgesetz (IfSG) zur kontinuierlichen Erfassung und Bewertung von Infektionen und Infektionserregern verpflichtet sind, werden routinemäßige Surveillancedaten zum Vorkommen von Infektionen in Pflegeheimen in Deutschland bislang nicht erhoben. Es liegen somit nur Daten aus vereinzelten Erhebungen vor. Die zur Erfassung erforderlichen Infektionsdefinitionen sind zum Zweck der Surveillance und Infektionskontrolle Anfang der 1990er-Jahre von McGeer et al. [30] speziell für den Bereich der Altenpflege modifiziert worden. Die überwiegend symptombasierten Definitionen tragen dabei der Tatsache Rechnung, dass anders als im klinischen Bereich in Altenpflegeeinrichtungen meist keine laborchemische, radiologische oder mikrobiologische Diagnostik durchgeführt wird. Gleichzeitig werden physiologische Besonderheiten des alten Menschen berücksichtigt.

Jüngere Daten aus Deutschland, die im Rahmen der europäischen Erhebung „Healthcare-associated infections in longterm care facilities (HALT) “ im Jahr 2010 in 73 deutschen Altenpflegeheimen erfasst wurden, geben eine Prävalenz von 1,6\% bzw. - unter Anwendung modifizierter (strengerer) McGeer-Kriterien - eine Prävalenz von $0,79 \%$ für gesicherte Infektionen an. Dabei machen die Harnwegsinfektionen den weit überwiegenden Anteil der Infektionen aus [15]. Eine andere deutsche Studie hat im Zeitraum 2006/2007 in 4 Altenpflegeeinrichtungen eine Prävalenz von $6,8 \%$ für Pflegeheim-assoziierte Infektionen ermittelt; in der Gruppe der Schwerpflegebedürftigen (Pflegestufe III) lag die Prävalenz sogar bei 12,3\%. Auch hier wurden vor allem Atemwegsinfekte, Haut-/Weichgewebsinfekte sowie Harnwegsinfekte dokumentiert [31]. In einer prospektiven Surveillance-Studie zur Inzidenz von Infektionen in 7 deutschen Pflegeheimen wurde im Zeitraum 2008/2009 bei 511 Bewohnern eine Gesamtinzidenz von 5,3 Infektionen pro 1000 Bewohnertage ermittelt. Infekte des Gastrointestinaltrakts, akute Atemwegsinfekte sowie Harnwegsinfekte waren hier die am häufigsten dokumentierten Infekte; viele traten allerdings im Rahmen von Clustern mit bis zu 17 betroffenen Bewoh- 
Tab. 4 Prävalenzstudien zum Vorkommen von Vancomycin-resistenten Enterococcus spp. (VRE) in Langzeitpflegeeinrichtungen

\begin{tabular}{|c|c|c|c|c|c|c|c|c|}
\hline \multirow[t]{2}{*}{ Autoren } & \multirow[t]{2}{*}{ Jahr } & \multirow[t]{2}{*}{ Nation } & \multirow[t]{2}{*}{ Studiendesign } & \multirow[t]{2}{*}{ Probenmaterial } & \multirow{2}{*}{$\begin{array}{l}\text { Anzahl } \\
\text { der Heime }\end{array}$} & \multirow{2}{*}{$\begin{array}{l}\text { Anzahl der unter- } \\
\text { suchten Bewohner }\end{array}$} & \multicolumn{2}{|c|}{ Prävalenz } \\
\hline & & & & & & & (\%) & $\mathrm{n}$ \\
\hline March et al. [45] & 2009 & Italien & Punktprävalenzstudie & $\begin{array}{l}\text { Rektale, inguinale, oropharyngea- } \\
\text { le, nasale Tupferabstriche, Urin }\end{array}$ & 1 & 111 & 2,7 & 3 \\
\hline Trick et al. & 2001 & USA & Punktprävalenzstudie & Rektaltupfer & 1 & 114 & 3,5 & 4 \\
\hline Stuart et al. & 2011 & Australien & Punktprävalenzstudie & Stuhlproben & 3 & 119 & 1,7 & 2 \\
\hline Padiglione et al. & 2001 & Australien & Punktprävalenzstudie & Stuhlproben & 8 & 292 & 3,1 & 9 \\
\hline Wendt et al. [44] & 1999 & $\begin{array}{l}\text { Deutsch- } \\
\text { land }\end{array}$ & Querschnittsstudie & Rektaltupfer & 1 & 188 & 4,3 & 8 \\
\hline Benenson et al. [46] & 2009 & Israel & Querschnittsstudie & Stuhlproben & 9 & 802 & 9,6 & 77 \\
\hline
\end{tabular}

Tab. 5 Prävalenzstudien zum Vorkommen von multiresistenten gramnegativen Bakterien (z. B. ESBL-bildende Bakterien) in

Langzeitpflegeeinrichtungen

\begin{tabular}{|c|c|c|c|c|c|c|c|c|}
\hline \multirow[t]{2}{*}{ Autoren } & \multirow[t]{2}{*}{ Jahr } & \multirow[t]{2}{*}{ Nation } & \multirow[t]{2}{*}{ Studiendesign } & \multirow[t]{2}{*}{ Probenmaterial } & \multirow{2}{*}{$\begin{array}{l}\text { Anzahl der } \\
\text { Heime }\end{array}$} & \multirow{2}{*}{$\begin{array}{l}\text { Anzahl der unter- } \\
\text { suchten Bewohner }\end{array}$} & \multicolumn{2}{|c|}{ Prävalenz } \\
\hline & & & & & & & (\%) & $\mathbf{n}$ \\
\hline O'Fallon et al. [54] & 2009 & USA & Punktprävalenzstudie & Rektaltupfer, Nasentupfer & 1 & 161 & 22,8 & 37 \\
\hline March et al. [45] & 2009 & Italien & Punktprävalenzstudie & $\begin{array}{l}\text { Urin, Rektaltupfer, Leisten- } \\
\text { tupfer, Nasen-Rachen-Tupfer }\end{array}$ & 1 & 111 & 64 & 71 \\
\hline Rooney et al. [47] & 2009 & Irland & Punktprävalenzstudie & Stuhlproben & 16 & 294 & 40,5 & 119 \\
\hline Pop-Vicas et al. [48] & 2008 & USA & Querschnittsstudie & Rektaltupfer, Nasentupfer & 1 & 84 & 51 & 43 \\
\hline O'Fallon et al. & 2010 & USA & Prospektive Kohortenstudie & Rektaltupfer & 1 & 172 & 31 & 53 \\
\hline Stuart et al. & 2011 & Australien & Punktprävalenzstudie & Stuhlproben & 3 & 119 & 12 & 14 \\
\hline Trick et al. & 2001 & USA & Punktprävalenzstudie & Rektaltupfer & 1 & 114 & 33 & 37 \\
\hline
\end{tabular}

nern in einer Einrichtung auf [32]. Diese Studie hat insbesondere die große Bedeutung der Surveillance für das Aufdecken und Erkennen epidemischer Zusammenhänge in Altenpflegeeinrichtungen verdeutlicht. Eine ähnliche Infektionsinzidenz (6,0 pro 1000 Bewohnertage) wurde auch in einer früheren prospektiven Surveillance-Studie ermittelt, die allerdings nur 1 Heim einschloss [17]. Erhebungen aus anderen europäischen Ländern zeigen teils etwas höhere Prävalenzen für Infektionen in Pflegeheimen: In Norwegen wurden Prävalenzen von 6,6-7,6\% ermittelt, in Italien $8,4 \%$ und in einer jüngeren Erhebung in Frankreich eine Gesamtprävalenz von $11,23 \%$ bei immerhin knapp 45.000 untersuchten Altenheimbewohnern [33, 34, 35]. Das Trio der Harnwegs-, Atemwegs- und Haut-/Weichgewebsinfektionen ist auch in diesen Erhebungen bei den dokumentierten Infektionen maßgeblich vertreten. In Erhebungen aus den USA rangiert die Inzidenz von Infektionen bei Pflegeheimbewohnern zwischen 1,8 und 13,5 Infektionen pro 1000 Bewohnertage [29].

Infektionsausbrüche in Altenpflegeeinrichtungen haben einen großen Einfluss auf die Morbidität und Letalität der Bewohner. Nicht selten zeichnen sie sich durch hohe Attack-Raten der Erreger und hohe Mortalitätsraten aus [18]. Bei den epidemisch auftretenden Infektionen überwiegen solche des Respirations- und Gastrointestinaltrakts bei Weitem. Sie sind in der Regel virusassoziiert. Die meisten publizierten Ausbruchsanalysen in Langzeitpflegeeinrichtungen berichten über Ereignisse durch Influenzaviren bzw. Noroviren. Als bakterielle Erreger kommen bei respiratorischen Ausbrüchen vorwiegend Pneumokokken, Mycobacterium tuberculosis und auch Legionellen vor. Bei den gastrointestinalen Ausbrüchen sind es vor allem Salmonellen, Shigellen, S. aureus, Clostridien und darmpathogene E. coli [36].

\section{Bedeutung antibiotikaresistenter Bakterien bei alten Menschen in Pflegeeinrichtungen}

Aufgrund der häufigen Verlegung zwischen Krankenhaus und Pflegeheim ergeben sich für die Bewohner von Altenheimen Risiken für die Kolonisation und Infektion mit multiresistenten Bakterien wie MRSA, Vancomycin-resistenten Enterokokken (VRE) oder Extended-
Spectrum- $\beta$-Lactamase (ESBL)-bildenden gramnegativen Erregern. Epidemiologische Studien, die sich mit der Kolonisation von Altenheimbewohnern mit mehrfach-resistenten Erregern beschäftigen, sind erst in der jüngeren Vergangenheit initiiert worden und haben sich meist auf MRSA oder VRE fokussiert. Für MRSA bei deutschen Altenheimbewohnern sind teils relativ niedrige Kolonisationsraten zwischen $1,1 \%$ und $3 \%$, teils aber auch höhere Prävalenzen von $7,6 \%$ veröffentlicht $[37,38,39,40]$. Genetische Analysen zeigen, dass es sich dabei in der Regel um Stämme handelt, die auch in Krankenhäusern prävalent sind [41]. Internationale Daten geben Prävalenzen von $0,33 \%$ in den Niederlanden bis hin $\mathrm{zu} 52 \%$ in den USA an $[42,43]$. Auch für VRE bei Altenheimbewohnern sind international Prävalenzen zwischen ca. $2 \%$ und knapp $10 \%$ beschrieben [44, $45,46]$. Die bislang publizierten Daten zu Prävalenzen beider Erreger sind in - Tab. 3 und 4 dargestellt.

Neuere Studien geben Hinweis darauf, dass eine Kolonisation mit mehrfach-resistenten gramnegativen Bakterien bei Bewohnern von Altenheimen häufig festzustellen ist [20]. Meist sind in 
diesem Zusammenhang vor allem ESBLproduzierende E. coli und K. pneumoniae beschrieben, deren Prävalenz in einigen europäischen Studien bereits zwischen $40 \%$ und $64 \%$ liegt $[45,47]$. $\bullet$ Tab. 5 gibt eine Übersicht über international publizierte Prävalenzraten für multiresistente gramnegative Bakterien in Pflegeheimen. Einige Studien konnten zeigen, dass eine gleichzeitige Besiedlung mit verschiedenen resistenten Bakterien nicht selten ist. Dies lässt sich nicht zuletzt auch durch ähnliche Risikofaktoren für eine solche Besiedlung erklären. $\mathrm{Zu}$ den bekannten Risikofaktoren für die Kolonisation mit mehrfach-resistenten Bakterien zählen z. B. ein Lebensalter von über 65 Jahren, eine antibiotische Behandlung innerhalb der letzten 3 Monate (insbesondere Cephalosporine und Fluorchinolone), Demenz, eine fäkale Inkontinenz und ein Lebensmittelpunkt in Altenheimen [45, 47, 48]. Für ESBL-verursachte Harnwegsinfekte bei nicht hospitalisierten Patienten wurden in einer israelischen Studie als Risikofaktoren eine Antibiotikatherapie innerhalb der letzten 3 Monate (vor allem mit Cephalosporinen, Chinolonen und Penicillinen), ein stationärer Krankenhausaufenthalt im selben Zeitraum, ein Lebensalter von über 60 Jahren, Diabetes mellitus sowie männliches Geschlecht identifiziert [49].

Allerdings ist bislang die Bedeutung und Konsequenz einer Besiedlung mit multiresistenten Erregern bei Altenheimbewohnern nicht ausreichend geklärt. Alle Spezies sind prinzipiell Bestandteile der physiologischen Körperflora und zeichnen sich primär durch das erweiterte Resistenzspektrum aus, nicht aber durch eine veränderte Virulenz oder Pathogenität. Unumstritten ist das erhöhte Infektionsrisiko im Fall von Krankenhausaufenthalten bzw. bei invasiven Eingriffen und Operationen [50, 51]. Jedoch scheint sich bei "gesunden“ alten Menschen, die mit resistenten Bakterien besiedelt sind und im Altenheim als ihrem häuslichen Lebensumfeld verbleiben, das Risiko für Infektionen nicht zwangsläufig allein durch die Kolonisation zu erhöhen. Infektionen - z. B. durch MRSA - kommen trotz der teils hohen Kolonisationsraten nicht häufiger in Altenheimen vor [52].

\section{Einsatz von Antibiotika in Pflegeheimen}

In den vergangenen Jahren hat sich bei der Verordnung von Antibiotika im Heimbereich eine Verschiebung hin zu den Chinolonen sowie zur dritten Generation von Cephalosporinen vollzogen [15]. Daten des deutschen Resistenzatlas GERMAP aus dem Jahr 2010 geben für den Zeitraum von 2003 bis 2008 einen Zuwachs im ambulanten Verordnungsvolumen von 34\% bei den Chinolonen und von $62 \%$ bei den Oralcephalosporinen an [53]. Besonders deutlich wird dies bei älteren Menschen. Ab dem 60. Lebensjahr werden Fluorchinolone bereits häufiger eingesetzt als neuere Makrolide. Sie stehen nach den Tetrazyklinen und Penicillinen bereits an dritter Stelle bei den Verordnungen. Ab dem 80. Lebensjahr werden Fluorchinolone als häufigste Antibiotikaklasse eingesetzt. Ergebnisse der europäischen HALT-Studie ergaben bei den Bewohnern deutscher Heime insgesamt eine Prävalenz für eine Antibiotikaverordnung von 1,15. Auch hier wurden vorwiegend $\beta$-Lactame $(33,8 \%)$ und Fluorchinolone (31,4\%) eingesetzt [15]. In den deutschen Heimen der HALT-Studie wurden $67,3 \%$ aller bei den Bewohnern auftretenden Infektionen mit oralen Antibiotika behandelt.

Betrachtet man die Daten aller 21 teilnehmenden Länder, die im Rahmen der HALT-Studie ermittelt wurden, stehen die Chinolone bei der empirischen Therapie von Infektionen an erster Stelle. Angesichts der voranschreitenden Resistenzentwicklung und des Selektionsdrucks, der durch den Einsatz dieser Antibiotikaklasse sowohl auf MRSA als auch auf die ESBL-Bildner ausgeübt wird, zeigt sich anhand dieser aktuellen Daten ein deutlicher Handlungsbedarf. Die Zunahme von Resistenzen gegenüber Chinolonen konnten O'Fallon et al. [54] bereits vor einigen Jahren anschaulich demonstrieren. In allen klinischen Proben eines sehr großen Pflegeheims, die im Zeitraum von 2003 bis 2005 auf das Vorkommen resistenter Bakterien analysiert wurden, stieg der Anteil an multiresistenten gramnegativen Bakterien von 7\% im Jahr 2003 auf $13 \%$ im Jahr 2005. 80\% dieser Isolate waren bereits resistent gegenüber Ciprofloxacin.
Kürzlich wurden auch Daten zur Indikation des Antibiotikaeinsatzes in allen Teilnehmerstaaten der HALT-Studie veröffentlicht. Der überwiegende Teil der Antibiotika $(54,4 \%)$ wird empirisch verordnet, und zwar am häufigsten zur Therapie von Atemwegsinfekten. Immerhin 28,8\% der verordneten Antibiotika wurden in dieser Erhebung „prophylaktisch“ eingesetzt, vor allem zur Prophylaxe von Harnwegsinfektionen [55].

\section{Wie ist es um die medizinische Versorgung von alten Menschen in Heimen bestellt, die mit multiresistenten Erregern besiedelt oder infiziert sind?}

Die Kommission für Krankenhaushygiene und Infektionsprävention (KRINKO) hat sich in der Empfehlung zur „Infektionsprävention in Heimen" ausführlich zum Umgang mit Erregern mit besonderen Eigenschaften, wie z. B. mit multiresistenten Bakterien geäußert. Es wird unabhängig von der Erregerspezies ein risikobasiertes Vorgehen unter Berücksichtigung der Verhältnismäßigkeit zwischen infektionspräventiven Maßnahmen und den Freiheiten des betroffenen Bewohners empfohlen. Die situationsabhängige Risikoeinschätzung vor Ort kann dabei nur in enger Zusammenarbeit zwischen betreuenden Hausärzten und Pflegepersonal erfolgen. Die Erfahrung zeigt jedoch, dass die Empfehlung der KRINKO vielen Heimen bzw. den zuständigen Pflegedienstleitungen nicht bekannt ist. Nicht selten ist in den Arbeitsanweisungen der Heime zum Umgang mit MRSAkolonisierten Bewohnern eine strikte Einzelzimmerunterbringung formuliert. Für die betroffenen Heimbewohner kann dies mitunter schwerwiegende Konsequenzen haben, insbesondere dann, wenn Sanierungsbehandlungen nicht erfolgreich sind und sich die Isolierungsmaßnahmen über einen längeren Zeitraum erstrecken. Isolierung kann bei dementen Patienten $\mathrm{zu}$ einem schnellen weiteren Verlust der noch vorhandenen geistigen Fähigkeiten führen [56].

Ebenso ist ein oft ratloses und zufälliges Vorgehen bei der MRSA-Sanierung zu beobachten. Erfahrungen der Autoren im Rahmen einer Studie bei 400 Heim- 
bewohnern in Berlin zeigen, dass die Entscheidung über Art und Ablauf einer Sanierungsbehandlung nicht selten allein beim Pflegepersonal liegt. Häufig wird die Sanierung auf die Anwendung von $\mathrm{Mu}$ pirocin-Nasensalbe beschränkt, obwohl z. B. der Nachweis von MRSA explizit aus der Leistenregion erfolgte. Dies ist nicht nur für die Bewohner, die nicht erfolgreich saniert werden, bedauerlich, sondern auch im Hinblick auf die zu erwartende Resistenzentwicklung von MRSA gegenüber Mupirocin alarmierend. Neben der Sanierung, ist oftmals auch die Dokumentation der durchgeführten Sanierungsbehandlung ungenügend und geht nicht selten in einer Fülle anderer Bemerkungen in den Bewohnerakten unter.

Auch im Umgang mit ESBL-bildenden Bakterien in Heimen lässt sich Informationsbedarf feststellen. Die „Heimempfehlung“ der KRINKO zielt auf eine Abstraktion der zu erstellenden Risikoprofile und Maßnahmenkataloge unabhängig von der Spezies des resistenten Erregers $\mathrm{ab}$, die jedoch von den Pflegedienstleitungen vielfach nicht geleistet werden kann. Hier entsteht mit jedem Befund oftmals eine erneute Verunsicherung zum Umgang mit kolonisierten Bewohnern. Der überwiegende Teil der Pflegedienstleitungen gab im Rahmen der oben erwähnten Studie der Autoren an, Bewohner, die mit ESBL-bildenden Bakterien besiedelt sind, bekämen in ihrer Einrichtung routinemäßig eine orale antibiotische Therapie durch den Hausarzt bzw. MupirocinNasensalbe oder antiseptische Körperwaschungen zur Sanierung der ESBL-Kolonisation.

\section{Welche Konsequenzen ergeben sich für die Zukunft?}

Nach diesen Beobachtungen lässt sich feststellen, dass insbesondere im Umgang mit besiedelten oder infizierten Bewohnern in Altenpflegeheimen sowie bei der Sanierung von MRSA-kolonisierten Bewohnern und der Dokumentation der Sanierungsmaßnahmen noch immer großer Handlungsbedarf besteht. Obwohl die geschilderten Probleme schon seit einigen Jahren beobachtet werden, und vielfach bereits die stärkere Einbindung des öffentlichen Gesundheitsdienstes in
Form von gezielten Fortbildungen und unterstützender Beratung gefordert wurde, besteht an der Basis, also bei den gelernten und ungelernten Pflegenden, die die Versorgung der alten Menschen übernehmen, nach wie vor ein großes Verbesserungspotenzial. Zumindest im Bereich der MRSA-Sanierung von Heimbewohnern, die nach einem stationären Krankenhausaufenthalt im Heim weiter betreut werden, kann im Zuge der kürzlich vom Bewertungsausschuss formulierten Vergütungsvereinbarung für ärztliche Leistungen zur Diagnostik und ambulanten Eradikationstherapie von MRSA-Trägern in der vertragsärztlichen Versorgung eine verbesserte Kooperation zwischen Heimleitung und betreuenden Hausärzten erwartet werden.

Eine weitere Stärkung der regionalen MRE-Netzwerke, die sich erfreulicherweise inzwischen in allen deutschen Bundesländern etabliert haben, ist dabei hilfreich, jedoch allein sicher nicht ausreichend. Auch die Kooperation zwischen behandelnden Ärzten sowie zwischen Ärzten und Pflegepersonal in den Heimen ist schon lange als Konvergenzpunkt für langfristige Erfolge in der Infektionsprävention und im Umgang mit resistenten Erregern bekannt, und dennoch weiterhin optimierungsbedürftig. Die Dokumentation des Antibiotikaeinsatzes in den Einrichtungen sollte vereinheitlicht und für alle Beteiligten nachvollziehbar und schnell verfügbar sein. Aufgrund der unklaren Symptomatik bzw. atypischen Präsentation von Infekten bei alten Menschen sowie der teils erschwerten mikrobiologischen Diagnostik ist die empirische Antibiotikatherapie in den Heimen oft nicht vermeidbar. Sie sollte dann jedoch auf einer guten klinischen Diagnose und unter Kenntnis der mikrobiologischen Epidemiologie vor Ort erfolgen. Grundlage hierfür schaffen die Implementierung einfacher Surveillancemodule, das Führen von Resistenzstatistiken sowie der intensive Austausch der behandelnden Ärzte unter Beteiligung des Pflegepersonals.

Im Zuge der Novellierung des Infektionsschutzgesetzes im vergangenen Sommer wurde dem Bereich der antimikrobiellen Therapie von Infektionen vom Gesetzgeber stärkere Beachtung geschenkt.
Die am RKI zu etablierende Kommission "Antiinfektiva, Resistenz und Therapie“ (ART) wird ihren Beitrag zur Optimierung der Diagnostik und Therapie von Infektionen, insbesondere solcher durch mehrfachresistente Problemerreger leisten. Die Implementierung der Empfehlungen vor Ort, insbesondere in Einrichtungen wie Altenpflegeheimen, wird weiterhin eine große Herausforderung für alle beteiligten Akteure sein und erfordert neben dem guten Willen vor allem gezielte Maßnahmen, die auch den scheinbar unvermeidbaren Charakteristika von Heimen wie ständigem Personalwechsel und immensem Arbeitsdruck gerecht werden.

\section{Korrespondenzadresse}

\section{Dr. C. Ruscher}

Abteilung für Infektionskrankheiten, Fachgebiet 14 (Angewandte Infektions- und Krankenhaushygiene), Robert Koch-Institut Nordufer 20, 13353 Berlin

ruscherc@rki.de

Danksagung. Besonderer Dank gilt Herrn Dr. Alfred Nassauer für die kritische und konstruktive Korrektur des Manuskripts.

Interessenkonflikt. Der korrespondierende Autor gibt für sich und seine Koautoren an, dass kein Interessenkonflikt besteht.

\section{Literatur}

1. Statistisches Bundesamt (2010) Demografischer Wandel, Auswirkungen auf Krankenhausbehandlungen und Pflegebedürftige im Bund und in den Ländern. Statistisches Bundesamt, Wiesbaden

2. Statistisches Bundesamt, Pfaff H (2011) Pflegestatistik 2009 Pflege im Rahmen der Pflegeversicherung Deutschlandergebnisse. In: Pflegestatistik. Statistisches Bundesamt, Wiesbaden

3. Ramroth H, Specht-Leible N, König H-H et al (2006) Use of hospital based resources by individuals in residential care homes. Dtsch Arztebl Int 103:2710-2713

4. Specht-Leible N, Bender M, Oster P (2003) Causes for in-hospital treatment of nursing home residents. Z Gerontol Geriatr 36:274-279

5. Castle SC (2000) Clinical relevance of age-related immune dysfunction. Clin Infect Dis 31:578-585

6. Ben-Yehuda A, Weksler ME (1992) Host resistance and the immune system. Clin Geriatr Med 8:701711

7. Yoshikawa TT (2000) Epidemiology and unique aspects of aging and infectious diseases. Clin Infect Dis 30:931-933

8. Meyer KC (2001) The role of immunity in susceptibility to respiratory infection in the aging lung. Respir Physiol 128:23-31

9. Nicolle LE (2000) Urinary tract infection in longterm-care facility residents. Clin Infect Dis 31:757761 
10. Levkoff SE, Marcantonio ER (1994) Delirium: a major diagnostic and therapeutic challenge for clinicians caring for the elderly. Compr Ther 20:550-557

11. Yoshikawa TT (1997) Perspective: aging and infectious diseases: past, present, and future. J Infect Dis 176:1053-1057

12. Yoshikawa TT, Norman DC (1996) Approach to fever and infection in the nursing home. J Am Geriatr Soc 44:74-82

13. Nicolle LE (2002) Urinary tract infection in geriatric and institutionalized patients. Curr Opin Urol 12:51-55

14. Marrie TJ (2000) Community-acquired pneumonia in the elderly. Clin Infect Dis 31:1066-1078

15. Wischnewski N, Mielke M, Wendt C (2011) Healthcare-associated infections in long-term care facilities. German results of the European prevalence study HALT. Bundesgesundheitsbl Gesundheitsforsch Gesundheitsschutz 54:1147-1152

16. Bradley SF (1999) Issues in the management of resistant bacteria in long-term-care facilities. Infect Control Hosp Epidemiol 20:362-366

17. Engelhart ST, Hanses-Derendorf L, Exner M, Kramer MH (2005) Prospective surveillance for healthcare-associated infections in German nursing home residents. J Hosp Infect 60:46-50

18. Strausbaugh LJ, Sukumar SR, Joseph CL (2003) Infectious disease outbreaks in nursing homes: an unappreciated hazard for frail elderly persons. Clin Infect Dis 36:870-876

19. Smith PW, Seip CW, Schaefer SC, Bell-Dixon C (2000) Microbiologic survey of long-term care facilities. Am J Infect Control 28:8-13

20. Nicolle LE (2002) Resistant pathogens in urinary tract infections. J Am Geriatr Soc 50:230-235

21. Kanellakopoulou K, Grammelis V, Baziaka F et al (2009) Bacterial flora in residents of long-term care facilities: a point prevalence study. J Hosp Infect 71:385-387

22. Mody L, Kauffman CA, Donabedian S et al (2008) Epidemiology of Staphylococcus aureus colonization in nursing home residents. Clin Infect Dis 46:1368-1373

23. Dommeti P, Wang L, Flannery EL et al (2011) Patterns of ciprofloxacin-resistant gram-negative bacteria colonization in nursing home residents. Infect Control Hosp Epidemiol 32:177-180

24. Gurwitz JH, Field TS, Avorn J et al (2000) Incidence and preventability of adverse drug events in nursing homes. Am J Med 109:87-94

25. Bernabei R, Gambassi G, Lapane K et al (1999) Characteristics of the SAGE database: a new resource for research on outcomes in long-term care. SAGE (Systematic Assessment of Geriatric drug use via Epidemiology) Study Group. J Gerontol A Biol Sci Med Sci 54:M25-33

26. Nicolle LE, Strausbaugh LJ, Garibaldi RA (1996) Infections and antibiotic resistance in nursing homes. Clin Microbiol Rev 9:1-17

27. Gavazzi G, Krause KH (2002) Ageing and infection. Lancet Infect Dis 2:659-666

28. Irvine PW, Van Buren N, Crossley K (1984) Causes for hospitalization of nursing home residents: the role of infection. J Am Geriatr Soc 32:103-107

29. Strausbaugh $L$, Joseph CL (2000) The burden of infection in long-term care. Infect Control Hosp Epidemiol 21:674-679

30. McGeer A, Campbell B, Emori TG et al (1991) Definitions of infection for surveillance in long-term care facilities. Am J Infect Control 19:1-7
31. Engelhart S, Lauer A, Simon A et al (2009) Repeated prevalence investigations of nursing home-associated infections as a tool to assess the hygienic quality of care. Bundesgesundheitsbl Gesundheitsforsch Gesundheitsschutz 52:936-944

32. Schulz M, Mielke M, Wischnewski N (2011) Clusters of infectious diseases in German nursing homes: observations from a prospective infection surveillance study, October 2008 to August 2009. Euro Surveill 16

33. Eriksen HM, Iversen BG, Aavitsland P (2004) Prevalence of nosocomial infections and use of antibiotics in long-term care facilities in Norway, 2002 and 2003. J Hosp Infect 57:316-320

34. Moro ML, Mongardi M, Marchi M, Taroni F (2007) Prevalence of long-term care acquired infections in nursing and residential homes in the Emilia-Romagna Region. Infection 35:250-255

35. Chami K, Gavazzi G, Carrat F et al (2011) Burden of infections among 44,869 elderly in nursing homes: a cross-sectional cluster nationwide survey. J Hosp Infect 79:254-259

36. Utsumi M, Makimoto K, Quroshi N, Ashida N (2010) Types of infectious outbreaks and their impact in elderly care facilities: a review of the literature. Age Ageing 39:299-305

37. Baum H von, Schmidt C, Svoboda D et al (2002) Risk factors for methicillin-resistant Staphylococcus aureus carriage in residents of German nursing homes. Infect Control Hosp Epidemiol 23:511-515

38. Woltering R, Hoffmann G, Daniels-Haardt I et al (2008) Prevalence of methicillin-resistant Staphylococcus aureus (MRSA) in patients in long-term care in hospitals, rehabilitation centers and nursing homes of a rural district in Germany. Dtsch Med Wochenschr 133:999-1003

39. Bock-Hensley O, Wendt C (2009) Methicillin-resistant Staphylococcus aureus in nursing homes. MMW Fortschr Med 151:41-45; quiz 46

40. Neuhaus B, Bocter N, Braulke Cet al (2002) Studie zum Vorkommen von Methicillin-resistenten Staphylococcus aureus in Alten- und Altenpflegeheimen in Nordrhein-Westfalen. Bundesgesundheitsbl Gesundheitsforsch Gesundheitsschutz 45:894-904

41. Pfingsten-Wurzburg S, Pieper DH, Bautsch W, Probst-Kepper M (2011) Prevalence and molecular epidemiology of meticillin-resistant Staphylococcus aureus in nursing home residents in northern Germany. J Hosp Infect 78:108-112

42. Reynolds C, Quan V, Kim D et al (2011) Methicillinresistant Staphylococcus aureus (MRSA) carriage in 10 nursing homes in Orange County, California. Infect Control Hosp Epidemiol 32:91-93

43. Greenland K, Rijnders MI, Mulders M et al (2011) Low prevalence of methicillin-resistant Staphylococcus aureus in Dutch nursing homes. J Am Geriatr Soc 59:768-769

44. Wendt C, Krause C, Xander LU et al (1999) Prevalence of colonization with vancomycin-resistant enterococci in various population groups in Berlin Germany. J Hosp Infect 42:193-200

45. March A, Aschbacher R, Dhanji H et al (2009) Colonization of residents and staff of a long-term-care facility and adjacent acute-care hospital geriatric unit by multiresistant bacteria. Clin Microbiol Infect

46. Benenson S, Cohen MJ, Block C et al (2009) Vancomycin-resistant enterococci in long-term care facilities. Infect Control Hosp Epidemiol 30:786-789
47. Rooney PJ, O'Leary MC, Loughrey AC et al (2009) Nursing homes as a reservoir of extended-spectrum beta-lactamase (ESBL)-producing ciprofloxacin-resistant Escherichia coli. J Antimicrob Chemother 64:635-641

48. Pop-Vicas A, Mitchell SL, Kandel R et al (2008) Multidrug-resistant gram-negative bacteria in a longterm care facility: prevalence and risk factors. J Am Geriatr Soc 56:1276-1280

49. Colodner R, Kometiani I, Chazan B, Raz R (2008) Risk factors for community-acquired urinary tract infection due to quinolone-resistant $E$. coli. Infection 36:41-45

50. Ben-Ami R, Schwaber MJ, Navon-Venezia S et al (2006) Influx of extended-spectrum beta-lactamase-producing enterobacteriaceae into the hospital. Clin Infect Dis 42:925-934

51. Safdar N, Maki DG (2002) The commonality of risk factors for nosocomial colonization and infection with antimicrobial-resistant Staphylococcus aureus, enterococcus, gram-negative bacilli, Clostridium difficile, and Candida. Ann Intern Med 136:834-844

52. Bradley SF (1997) Methicillin-resistant Staphylococcus aureus in nursing homes. Epidemiology, prevention and management. Drugs Aging 10:185-198

53. GERMAP (2010) Antibiotika-Resistenz und -Verbrauch. In: Bundesamt für Verbraucherschutz und Lebensmittelsicherheit; Paul-Ehrlich-Gesellschaft für Chemotherapie e.V.; Infektiologie Freiburg

54. O'Fallon E, Pop-Vicas A, D'Agata E (2009) The emerging threat of multidrug-resistant gram-negative organisms in long-term care facilities. J Gerontol A Biol Sci Med Sci 64:138-141

55. Latour K, Catry B, Broex E et al (2012) Indications for antimicrobial prescribing in European nursing homes: results from a point prevalence survey. Pharmacoepidemiol Drug Saf

56. McCusker J, Cole M, Dendukuri N et al (2001) Delirium in older medical inpatients and subsequent cognitive and functional status: a prospective study. CMAJ 165:575-583 